\title{
Cloud Gathering Model of Manufacturing Resources Based on P2P
}

\author{
Yunxia Jiang, Bowen Zhao, Dongnan Sun \\ School of Automation, Harbin Science and Technology University, Harbin, China \\ hrbustjyx@163.com
}

\begin{abstract}
Under the environment of cloud manufacturing, in view of the self-organization of manufacturing resources based on the ideas of the hybrid P2P and Chord agreement, three layer network models of manufacturing resources have been constructed. According to the structure of geographical area, the bottom centralized resources ring was built in each area; logic ring was structured by the gathering nodes, namely regional resources managers; logic main ring was formed by super nodes. And logic rings are DHT overlay, which are two layers of network. This paper expounds the dynamic organization way for manufacturing resources of two layers of DHT and the layered trust mechanism of the gathering network. In the end, the prototype instance of the resources cloud was presented.
\end{abstract}

Keywords: cloud manufacturing ; manufacturing resources ; gathering ; P2P

\section{Introduction}

With the development of information technology and cloud computing technology, fundamental changes have taken place in today's manufacturing environment, and its form is from traditional manufacturing to service. Makers tend to search for sharing and reasonable distribution method of manufacturing resources. Oriented to networked manufacturing, various enterprises as resources bearing point, perform the cooperation of different levels each other, and share the manufacturing application tasks, in order to realize the resource pooling and integration of manufacturing process [1]. Based on the thoughts of sharing resources in Internet, this loose collaboration needs the supports of the open and new information models and the architecture of resource search.

Under the manufacturing environment, the concept of cloud manufacturing was first presented by Chinese scholars, cloud manufacturing model highlights sharing ideas of the bottom resources of the cloud computing [2]. Through the cloud computing technology and related platform, it realizes to coordinate the equipment, materials, manpower, technology and information, and many other resources distributed in companies, to build an openly and dynamically changed, distributed and physical cloud pool, As well as a virtual pool. Base on the pool of shared resources, manufacturing environment of dynamic coordination has been structured, the underlying manufacturing resources have been optimally used [3].

In the manufacture cloud, manufacturing resources are self-organization and selfconvergence, and manufacturing resources cross-regionally and randomly exist. For service discovery and retrieval of resources, more consideration locates robustness, scalability, and equality of resource nodes [4]. As the carrier of distributed resources, Cloud manufacturing nodes loosely exist and couple together; the opportunity of manufacturing tasks for each load node is equal. Cloud manufacturing perfects its own structure by strengthening of gathering; and resource nodes freely comes into or goes outside from the network. 
Peer-to-peer (P2P) network model emphasizes the equal status of nodes of the system, and the flexible storage and utilization of resources, with the strong extensibility. It is suitable for the convergence model of manufacturing resources [5-8].

To accord with the organization model of manufacturing resources, this paper adopts the hybrid P2P network structure. Manufacturing resources are stored in local nodes, and adopt the centralized P2P network. Through DHT overlay network structure of two layers, the convergent synthesis for application is realized. The paper emphatically expounds the aggregation of manufacturing resources based on hybrid P2P structure, and the trust mechanism used in distributed resources is presented on the basis of the characteristics of the distributed network.

\section{Convergence Framework of Manufacturing Resources based on Hybrid P2P Network}

\subsection{The Basic Concept Model}

Cloud manufacturing is a new service mode in the networked manufacturing, is made by each element of the cloud mode and the environment of network manufacturing system. In the cloud manufacturing network, manufacturing resources and the virtual service are distributed. As shown in Figure 1, on the basis of the level thought of network nodes, the distributed resources information converges to center, and three logical nodes are defined. Load node (LN) is defined for the interface layer nodes of physical manufacturing resources, Load nodes are used to store the dynamic and static information of manufacturing resources, which are perceived and recognized. Aggregation node (AN) is defined for the management node of regional resources, owns the jurisdiction of load nodes, and is the nodes of carrying virtual resources, to store distributed virtual cloud pool of manufacturing resources, and to achieve the convergence between nodes and service center and the interaction between nodes. For application and design, Service center (SC) is defined for super gathering node, to provide support for manufacturing application, to carry category information of pooling resources, and to own the jurisdiction of the AN node. Gathering node and service center are collectively referred as the cloud nodes and the service nodes of cloud manufacturing, at the same time they also are physical unit of the cloud manufacturing network.

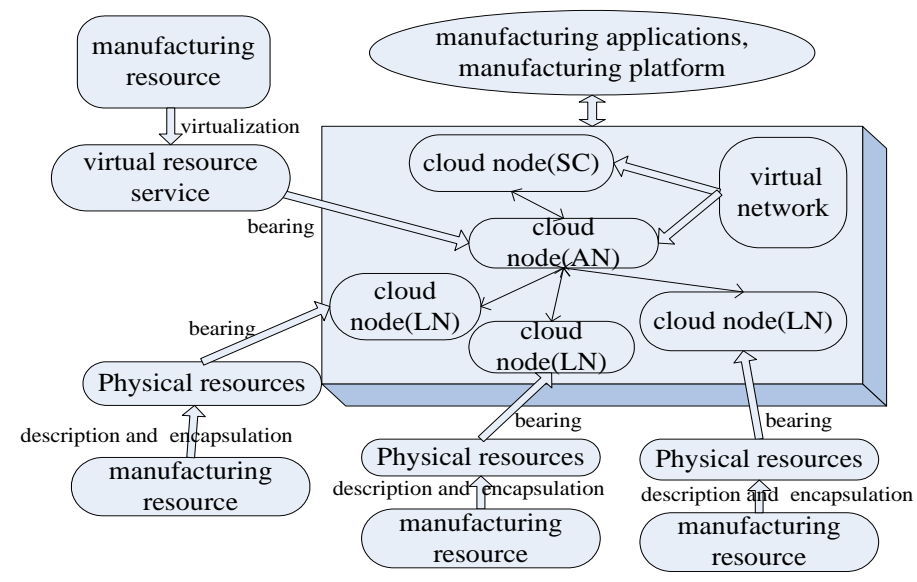

Figure 1. Concept Model of Cloud Manufacturing Resources 


\subsection{Convergence Structure of Manufacturing Resources}

The gather target of cloud manufacturing resources is the stable resource storage and retrieval, and builds the corresponding layer of abstraction. To the dynamic and distributed resources, the user can do preferred choice and don't need care about resource handling details. Logic level as shown in Figure 2, load node (LN) is at the bottom of converged network (layer 3) of the hybrid P2P resources network. AN is a load node of virtual resource to become the second floor of the converged resources network, and service center (SC) is the first layer of the network. The manufacturing resources randomly access $\mathrm{LN}$ in every region, and $\mathrm{LN}$ can manage local resources. At the same time a cloud droplet (cloud node) is formed on the basis of loose $\mathrm{LN}$ of regions, and AN can manage the cloud droplet of local LN, and run centralized P2P network platform to implement the centre index of the $\mathrm{P} 2 \mathrm{P}$ structure. But the loose cloud node executes the distributed P2P structure, by using the self-clustering model. Through the DHT overlay network structure, AN interacts with each other. SC is set as the super node of two layers of DHT to relay the information between the AN. Hybrid P2P networks reduce the flooding search of the network traffic, and realizes the free search and centralized management.

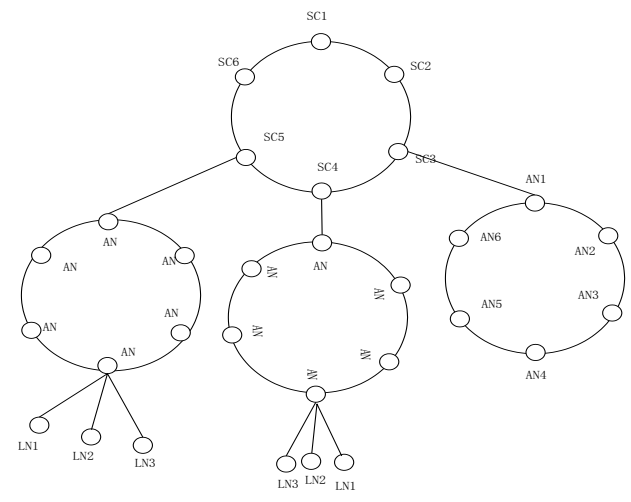

Figure 2. Convergence of Logic Level of Manufacturing Resources

As access interface of manufacturing resources, AN provides registration and retrieval of resources and routing information of LN. AN has center index library that is used to store the summary information of resources and the corresponding relation between resources and LN. AN dynamically requires update information of resources of $\mathrm{LN}$, to realize the updates of center index database. When AN needs some information of resources, the index table determines the node that owns the resources, to further obtain detailed information from LN. There are more than one LN that have the function of the AN, in order to realize the AN redundancy. According to the corresponding mapping rules, AN uses the information from $\mathrm{LN}$, to update the virtual resource pool. AN sends category information to super node SC in the loop on a regular, the category information, which is in the class library of resources in SC, could be updated automatically. SC is an application interface of the manufacturer and the starting point of the resources search. On the basis of manufacturing tasks, the platform of system initiates resource search from the AN and LN by SC. There are more than one AN, which have the function of the SC in AN ring, and virtual resource pool has been periodically synchronize between SC. But there can be only one current SC to work oriented application, other only play the role of the AN, as to support redundancy of manufacturing application, to improve the robustness of the system.

In Cloud manufacturing systems, cloud nodes are equal to each other, it could only manage a LN, may also manage a number of LN, without regard to the size of the distinction. Facing the common business opportunities between them, they are able to 
independently and coordinately finish manufacturing task, and its process is a trend from disorderly to orderly. Through the convergence, more members are constantly evolved and absorbed, and its own structure is also continuously improved, so the continuing business and adaptability of the market will be improved.

\section{Dynamic Organization of DHT Network of Manufacturing Resources}

There is large number of nodes in the network; therefore, the complexity of search is the main basis for selecting DHT network. This paper designs DHT network of two layers above $\mathrm{AN}$ and $\mathrm{SC}$ in the close to $\mathrm{O}(\log (\mathrm{N}))$ of Chord [9].

In the logic network of two layers of DHT, according to the nearby principle of physical location, AN (the second layer) consist some area rings, and every ring is a regional cluster. Based on the characteristics of the IPV4 addresses, according to the address structure of $\mathrm{C}$ class, each cluster is divided into a kind of Cord ring. SHA - 1 hash value of the first 24 bits of IP address is used as the regional identifier. In an area ring, a maximum number of junction points are 254. Each AN needs to maintain keys table of resources (Message)and three information, which are used to guarantee routing and stability of AN network: information of front node, list $\mathrm{L}$ of back nodes and pointer table (Finger Table), the data structure of Message as shown in table 1 (a). Key values of virtual resource service are derived from the service attribute key parameter set, corresponding to the user demand parameters.

SC (super node) consists upper layer (layer 1), which is a logical ring of Cord; and SC (super node) come from the node of AN ring. In order to simplify the build of SC logic ring, regional identity ANID is used as ID value of SC on the logical ring. The mechanism of query and routing of SC is similar to the AN annulus. The Message list as shown in table 1 (b), types of resources are classified induction of the AN ring, saved in type tree of Message, and marked by the tree from the root to the leaves, in order to the regional search of resources.

P2P network of multistage structure has strong expansibility, in the resources network key points are the network resources of nodes, rather than the node. According to the matching rules, search parameters are derived, through user requirements. According to the size of the contribution in the process of search, the search parameters are ordered. On $\mathrm{SC}$ ring, on the basis of the main parameters and the chord routing protocol, the type key of resources can be acquired. The next step is to lock regional clusters of AN, combining resource type tree. In AN area cluster, through the comparison of parametric and key value of virtual resource service, the instance set of resources will be found, to achieve the search of AN ring. The optimum matching of resource instances will be realized, by AN index of resources library.

Table 1 (a) Message Table of AN Node

\begin{tabular}{|c|c|c|}
\hline $\begin{array}{c}\text { regional } \\
\text { identifier(ANID) }\end{array}$ & $\begin{array}{c}\text { Key value set of virtual resource service } \\
(\text { key1, key2, key3......) }\end{array}$ & $\begin{array}{c}\text { super node identifier } \\
\text { (SUPERID) }\end{array}$ \\
\hline
\end{tabular}

Table 1 (b) Message Table of SC Nodes

\begin{tabular}{|l|l|l|}
\hline regional identifier ANID & topology of resource type tree & ID of entrance of AN ring \\
\hline
\end{tabular}

\subsection{Node Access}

Liking all $\mathrm{P} 2 \mathrm{P}$ system, to a new node, the joining method of $\mathrm{AN}$ or $\mathrm{SC}$ is the node access algorithm of Cord. It needs to contact a configuration node, to gain the initialization information, and to send messages to update the routing information of the 
other nodes in the network, so other nodes in the overlay network can update their status table. However, in DHT network of two layers, as a link of the AN cluster nodes, SC needs to solve the associated problems between AN and SC, therefore, when any node (assuming that is $\mathrm{n}$ ) wants to join, two things will be considered: 1) which of AN ring will the node $n$ belongs to? How joins into the corresponding area? 2) $n$ has the ability of SC or not; if there be, the ability of $n$ will be equal to super node of $\mathrm{AN}$ in the cluster. If its quantity and quality are all right, $\mathrm{n}$ will be become SC.

For $\mathrm{n}$ adding to a cluster, SC become a configuration index node, and will be obtained by looking for region labeling. Here, on SC ring, the configuration node is defined as the node agent that must be the nodes of well-known address. Specific algorithm is as follows:

Step 1. Calculate the IP address of n; acquire region labeling and identifier (assuming that

Step 2. Through a proxy node, for keyword of SC ring, $\mathrm{m}$ is come up to obtain the index of $\mathrm{SCm}$ that has jurisdiction over $\mathrm{m}$.

Step 3. If SCm exists, AN ring entry IDm will be obtained, into step 4; If SCm doesn't exist, an AN clusters that has a new logo m will be established; and $\mathrm{n}$ is set to $\mathrm{SCm}$, into the join algorithm of super node.

Step 4. If IDm is as configuration node ID, according to the join algorithm of Cord, it is able to join an AN cluster.

Step 5. The ability of $n$ is assessed. If $n$ has the ability to be a super node, super node selection algorithm will be started. If it is better than $\mathrm{SCm}, \mathrm{SCm}$ substitution algorithm will be executed; $\mathrm{n}$ will be set for the new super node, and the original $\mathrm{SCm}$ changes into backup node; the algorithm is over. If the quantity of SC does not exceed the threshold value of SC, $\mathrm{n}$ is SC, the algorithm is over; otherwise, $\mathrm{n}$ is a backup node, the algorithm is over.

\subsection{Node Exit and Failure}

The node can freely join or quit form the ring, but it is required to initiatively send messages of exiting, so the other nodes can immediately update their status table, to ensure dynamic adaptability of the overlay network. When an AN wants to leave from overlay network, it takes the initiative to tell other nodes to replicate the index information of resource in area cluster, other nodes will immediately update their own hash table, to ensure the efficiency of message routing. If SC exits, it needs to start the backup SC, in addition to execute the same exit steps of AN.

Based on the principle of Cord, if an AN fails, its precursor nodes will be rebinding by maintaining the subsequent list. When subsequent node fails, subsequent successor will be got out from the list for link connection. If SC node fails, a new SC will be competitively selected from backup nodes, and routing information can refresh itself. Because the node ID of SC is the regional identifier, on super node ring, other nodes will timely update ID and IP address.

\section{Trust Mechanism of Network}

Under the environment of P2P network, the equality of communication nodes is the common features of many application systems, and all nodes must be unconditional to obey the unified principle of organization and communication standards [10]. Trust mechanism of network has to inhibit privilege behavior, and guarantee the equality of all nodes. At the same time, in the application system, the quality of service is directly affected by the security and usability of resources. Common security solution of peer-topeer network environment is to build a trust mechanism of resource node. Resource applicants provide evaluation to resource providers after a transaction. The calculated value of the multiple evaluations is a trust. In the later, other resources applicants will use the trust as the reference to choose reliable resource provider. 
This article uses the hierarchical trust mechanism. In the local area, AN is a node owning higher credibility in LN, and its online time and storage capacity also reach the weights of standards. Through monitoring parameters of transaction records of LN, AN deletes LN whose trust is less than a certain threshold, and ensures that LN has high security in the concentrated area.

The security between the AN belong to the safety on the chord ring (the cluster area). Reputation value(RV) is adopted in the system to measure the security of the AN, RV is determined by the value of the weighted average of value itself and other comments in the routing table, The size of RV as the basis of choosing resource provider. The security of $\mathrm{SC}$ is as the safe value between cluster, and RV is the primary factor to choose the SC. The connected load rate of SC is defined as a percentage, namely the number of AN more than the capacity of SC. In order to ensure the safety of the SC, when the connection load rate is more than the threshold, the increasing SC algorithm will be started. On this basis of RV exceeding the threshold, measured weight algorithm will be considered, namely the SC selection algorithm, its parameters including resource category, online time and ability value, and so on. This method of hierarchical trust ensures the safe and reliable process of resource trading.

\section{Prototype Instance}

On the basis of the above research, cutting processing resources for instance, a prototype system of the gathering resources is exploited, interface shown in Figure 3. By using the cloud computing platform to simulate the network topology of distributed manufacturing resources, the gathering network of resources is built, which is hybrid P2P network of three layers. Each of virtual machines is the host of resources, to save a number of the database of Physical resources [11]. It simulates LN, and realizes the first layer of the prototype system, namely centralized P2P topology. On the basis of LN, the clusters (AN or SC) ring have been implemented, which are based on chord. Distributed virtual resource pools have been constructed in all $\mathrm{AN}$; the agreements of service and management are used in the communication between AN and LC, and the contact between $\mathrm{SC}$ and $\mathrm{AN}$.

The evaluation factors of AN include resource ownership, IT parameter and network performance in region of $\mathrm{LN}$, etc. In AN cluster, the condition of SC is selected, namely IT parameter, network performance, credibility, degree of regional belonging and so on. The method of dynamic determination uses the analytic hierarchy process, to judge the optimization of upper nodes through the weight [12]. For selecting and validating effect of different weight to optimize the upper node, the two kinds of weights, which are used to select SC, are calculated respectively, and the two kinds of optimal selection results as shown on Figure 4. The experiment proved that the different weights reflect the preference of different evaluation parameters, and logical nodes (AN or SC) can dynamically obtain in the network of three layer.
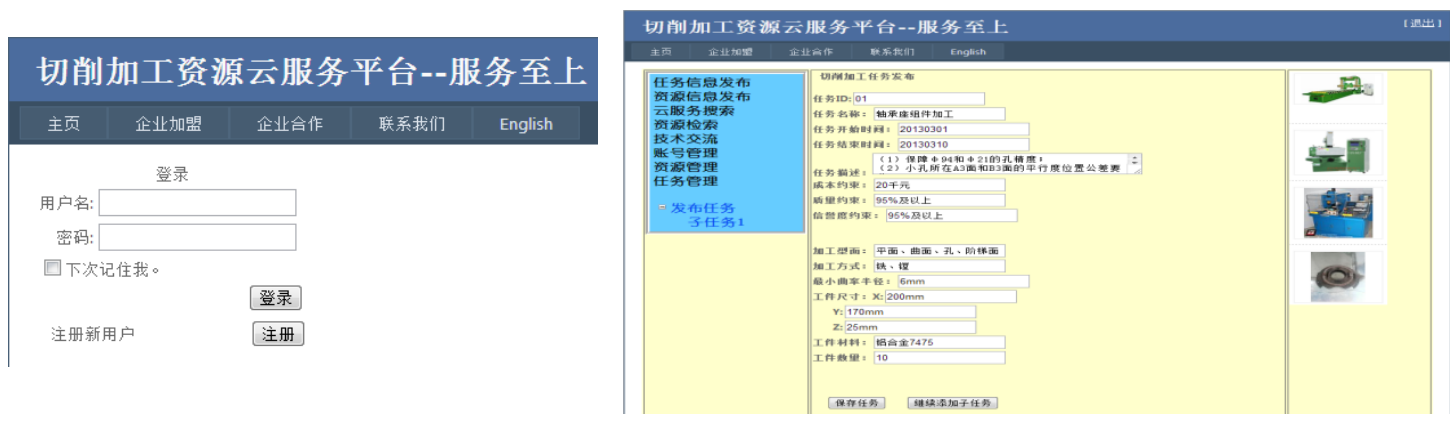

Figure 3. Management Interface of the System of Gathering Manufacturing Resources 

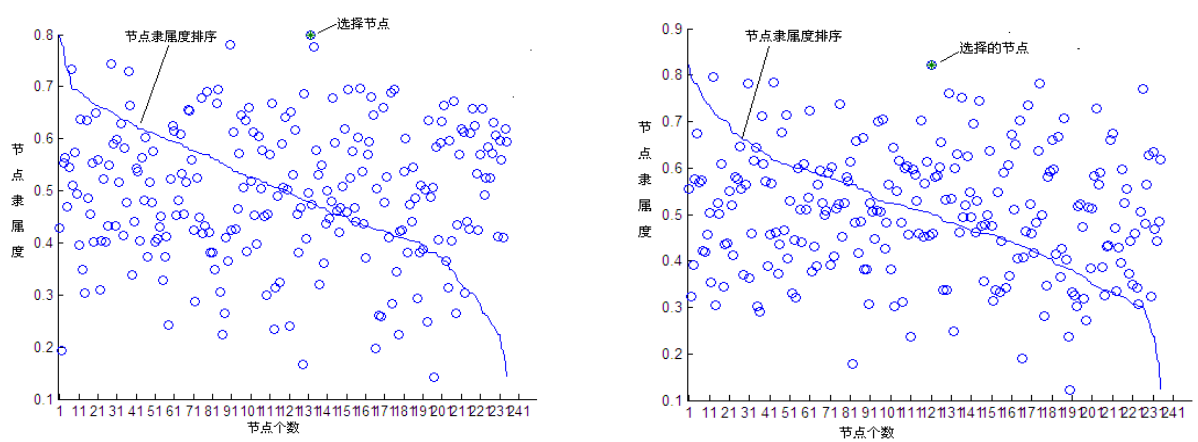

\section{Figure 4. Two Kinds of Result of Dynamically Selected Logical Nodes}

\section{Conclusions}

The paper proposes the gathering model of distributed manufacturing resources, based on a hybrid P2P network and Chord structure of two layers. This convergence model has realized with independent and loose coupling to some extent between the underlying resources and upper application. This paper expounds two layers network structure which is formed by the nodes of distributed manufacturing resources, designs and realizes the dynamic clustering structure and network trust mechanism. And on the basis of gathering model, gathering system of the cutting processing resources is built, at the same time management interface of the system is given. But the incentive mechanism of gathering resources needs to further research. In the future, with the gradually perfect cloud computing technology, efficiency and reliability of resource convergence will need further research.

\section{Acknowledgements}

This work is partly supported by the College Students' Innovative Experiment Funds for the Harbin Science and Technology University.

\section{References}

[1] T Fei, Z Lin, G Hua, et al. "Typical characteristics of cloud manufacturing and several key issues of cloud service composition”. J. Computer Integrated Manufacturing Systems, vol. 17, no. 3, (2011), pp. 477-486.

[2] Y Jiang, S Wang, M Zheng. "Research of Manufacturing Resource Sharing System Based on P2P”. J. International Journal of Grid and Distributed Computing, vol. 7, no. 5, (2014), pp. 275-284,

[3] T Dongming, L Xianliang, Y Lei. "Unstructured P2P search mechanism based on ant colony optimization". J. Advances in Information Sciences and Service Sciences, vol. 4, no. 11, (2012), pp. 257-263.

[4] BQ Huang, CH Li, C Yin, XP Zhao. "Cloud manufacturing service platform for small- and medium-sized enterprises". J. The International Journal of Advanced Manufacturing Technology, vol. 65, no. 9-12, (2013), pp. 1261- 1272.

[5] L Shijun, Q Benke, W Lei, M Xiangxu. "Research on gathered framework of self-organizing cloud manufacturing resources and searching method of multidimensional attribute range". J. Journal of computer aided design and graphics, vol. 24, no. 3, (2012), pp. 299-307.

[6] Alexopoulos, Kosmas, Makris, Sotiris. "Towards a role-centric and context-aware information distribution system for manufacturing". International Conference on Digital Enterprise Technology DET, Stuttgart, Germany, March (2014), pp. 377-384.

[7] D. G Zhang., Y. X Hu., D Wang.. "A new algorithm of service discovery based on DHT for mobile application".J. Journal of Network, vol. 6, no. 10, (2011), pp. 1466-1-474.

[8] X Tang, B Jiao. "Research on Inherent Physical Mechanism for the Impulsiveness of Aggregated Network Traffic". J. Journal of Harbin University of Science and Technology in China, vol. 16, no. 2, (2011), pp. 29-33.

[9] M Dong, M Maode, "Network selection and resource allocation for multicast in HetNets". J. Journal of Network and Computer Applications, vol. 43, no. 8, (2014), pp. 17-26. 
[10] B Xu, M Zhang, "The improved fuzzy analytic hierarchy process (ahp) applied in the evaluation of water quality of LinAnDuan of the south shao creek". J. Journal of environmental science, vol. 31, no. 9, (2011), pp. 2065- 2072.

[11] L Gao., Y. M Zhang., X Wu. "Improvement of Chord search algorithm research in P2P network". J. Application Research of Computers. vol. 31, no. 2, (2014). pp. 158-521.

[12] T Zhenhua, W Xingwei, Z Zhiliang. "The P2P distributed trust evaluation model based on multidimensional history vector”. J. Journal of computer, vol. 9, no. 33, (2010), pp. 1725-1735. 